\title{
Die Umsetzung hinkt
}

\author{
Die Rolle der Krankenpflege in der Primärversorgung
}

Die Primärversorgung erscheint vielen Gesundheitsverantwortlichen als Lösung der Probleme im Österreichischen Gesundheitssystem. Die Rolle und Kompetenzen der Gesundheits- und Krankenpflege sind in diesem Zusammenhang eher diffus angelegt, dabei stellt sie mit mehr als der Hälfte der im Gesundheitswesen tätigen Berufsangehörigen einen essentiellen Pfeiler der Versorgung dar. Pflege ist heute zwar besser sichtbar als noch vor 20 Jahren, die öffentliche Wahrnehmung von Gesundheits- und Krankenpflege in ihrer Kompetenz und Verantwortung ist jedoch immer noch schlecht beleuchtet. „Der Scheinwerfer gehört auf die Gesundheits- und Krankenpflege gerichtet", forderte denn auch Mag. Karl Schwaiger, Präsident des Verbands der Österreichischen Pflegedirektoren (ANDA), beim 8. Pflege-Management-Forum Anfang März in Wien.

Die Position der Pflege im Team, die Verzögerung der Reform des Gesundheitsund Krankenpflegegesetzes, die Rolle der Pflegewissenschaft, aber auch Qualitätssicherung und gesetzliche Rahmenbedingungen für die praktische Arbeit standen am Programm des hochkarätig besuchten Forums. Im kritischen Fokus stand da etwa die Neugestaltung der Primärversorgung in Österreich. Als einer der wesentlichen Bausteine der Gesundheitsreform, die im Jahr 2013 beschlossen und 2014 um das gesetzlich verankerte Bekenntnis zu eben dieser Primärversorgung ergänzt wurde, sollte dieser interdisziplinäre Zusammenschluss die überlasteten Krankenhäuser entlasten und damit eine effizientere Patientenversorgung sicherstellen. „Der Patient soll vor einer Fehl-, Unter- oder Überversorgung geschützt werden“, stellte Univ.-Prof. Dr. Manfred Mayer, Medizinische Universität Wien, fest und ergänzte: „Das Krankenhaus ist nicht immer nur ein Ort, wo man gesund wird“. Die Verlagerung der Patientenbetreuung in den ambulanten Bereich, wo dies aufgrund des Zustands des Patienten möglich ist, könnte diesen Schutz des Patienten deutlich verbessern. Indes - die breite Umsetzung scheiterte bislang.

\section{Der Wert der Leistung der Pflege}

Die Errichtung von Primary Healthcare Centers (PHC) wird von verschiedenen Interessenlagen behindert. $\mathrm{Ob}$ es darum geht, dass das Team "um den Hausarzt“ oder doch um den Patienten - gebildet wird, ob es um Öffnungszeiten, arbeitsrechtliche oder Kompetenzzuordnungen geht. Denn Primärversorgung, so Helmut Ivansits von der Arbeiterkammer Wien, sei mehr als die hausärztliche Versorgung und beinhalte auch pflegerische Leistungen. Hier sei jedoch nicht geklärt, was die Leistung der Pflege wert sei - nicht nur im Zusammenhang mit dem PHC, kritisierte Ursula Frohner, Präsidentin des Österreichischen Gesundheits- und Krankenpflegeverbands. Eine Finanzierungsreform sei derzeit nicht einmal angedacht und mit den Verantwortlichen auch nicht auf Augenhöhe verhandelbar.

Weiterhin ungeklärt ist die Einbindung der mobilen Dienste, ergänzte Mag. Monika Wild vom Österreichischen Roten Kreuz: „Es ist alles offen.“ Ebenso offen, wie die Frage, welche Rolle die Pflege zukünftig einnehmen sollte. „Das PHCGesetz ist als Anfang einer Reise zu verstehen", meinte Dr. Herwig Ostermann, Gesundheit Österreich GmbH, als Vertreter der Gesundheitsministeriumsseite. In der Umsetzung sei man derzeit nicht besonders stark, denn die Fragen „Wer macht was und wer bezahlt was" seien nach wie vor nicht beantwortet. Die mangelnde Umsetzungsstärke bestätigte Frohner und führte als zusätzliches Beispiel die stockende Reform des Gesundheits- und Krankenpflegegesetzes an, die für das Jahr 2015 erwartet worden war. Darin sollten unter anderem die Verordnungskompetenz für Medizinprodukte im Pflegebereich und die Bedarfsmedikation enthalten sein, aber: „Es fehlt am Mut der Politiker. Denn abseits von Standesinteressen wünschen sich alle Gesundheitsberufe die Adaptierung der Kompetenzen - und in der Praxis funktioniert es auch."

\section{Öffentlichkeit für die Pflege}

Als Gesundheitsminister war Alois Steger bereits Gast beim Pflege-Management-
Forum, diesmal beleuchtete er als Sozialminister die Anliegen der Gesundheits- und Krankenpflege und betonte deren Stellenwert und die Notwendigkeit der Öffentlichkeit für das Thema

Pflege. Es gehe, so Stöger, um die Stärkung der Professionalität, aber auch im die Verknüpfung von professioneller Pflege und häuslicher Pflege. Bessere Ausbildung und der reflektierte und unterstützte Umgang mit den täglichen Erfahrungen könnten dazu beitragen, die Berufsverweildauer zu heben, die aufgrund der hohen körperlichen und auch Beziehungsarbeit in diesem Beruf eher gering ist.

\section{Individualität braucht Mut}

In bewährt pointierter Weise setzte der Genetiker, Buchautor, stellvertretende Vorsitzende der österreichischen Bioethikkommission und Mitglied des Migrationsrates des österreichischen Innenministeriums sowie der Beratungsgruppe des österreichischen Finanzministers, Univ.-Prof. Dr. Markus Hengstschläger, den eloquenten Schlusspunkt zu seinem aktuellen Thema „Durchschnittsfalle“. Die Gene jedenfalls machen nur einen kleinen, wenn auch manchmal entscheidenden Teil der individuellen Unterschiede aus, stellte Hengstschläger fest. Es gehe darum, die Individualität zu entdecken und dort zu fördern, wo es eine genetische Voraussetzung dafür gibt. Dafür brauche es Ehrlichkeit auch sich selbst gegenüber, was man kann und was nicht. Denn: „Individualität braucht Mut!“

Über die procareScience Lecture von Dr. Eleonore Kemmtmüller und die Verleihung des cura 2016 - Pflege Management Award folgt in procare $4 / 2106$

Quelle: Pflege-Management-Forum 2016 3. - 4. 3. 2016, Wien 